\title{
Automatic Reading of Traffic Tickets
}

\author{
Nabeel Murshed \\ Intelligent Information Systems \\ A Pattern Recognition and Information Technology Company \\ Dubai, United Arab Emirates
}

\begin{abstract}
The present work presents a prototype system to extract and recognize handwritten information in a traffic ticket, and thereafter feeds them into a database of registered cars for further processing. Each extracted information consists either of handwritten isolated Arabic digits or tick mark "x". The ticket form is designed in such a way to facilitate the extraction process. For each input, the output of the recognition module is a probabilistic value that indicates the system confidence of the correct pattern class. If the probabilistic output is less than the determined threshold, the system requests assistance from the user to identify the input pattern. This feature is necessary in order to avoid feeding in wrong information to the database, such as associating the traffic ticket with the wrong registered car.
\end{abstract}

\section{Introduction}

Automatic reading of documents has become an interesting application area of Document Analysis Systems. Many systems have been developed for a wide range of applications. The reader is referred to the proceedings of the IAPR Workshops on Document Analysis Systems [1-4]. Most systems have been targeted towards Latin, Germanic, and Far Eastern languages. The present work is aimed at extracting and recognizing Arabic handwritten numerals for police application. To our knowledge, no system have been developed so far for such application.

Independent of the language, most automatic reading systems share two principle requirements: high recognition rate and short processing time, which are directly related to each other. In almost all cases, achieving high recognition rates could yield a relatively long processing time. One may argue that with the high speed of personal computers, one could obtain acceptable recognition results with acceptable processing time. A third important requirement is the degree of human interaction to increase the recognition rate, particularly in critical applications.

The objective of the proposed system is to extract and recognize six pieces of handwritten information from a traffic violation ticket. The information represent the following: date and time of violation, number and color of the number plate, violation type, and the policeman's ID number. All of those information are handwritten numerals except the color and violation type which are tick mark " $x$ ". The recognized information are put into a record associated with the number plate. Figure 1, in page 4 , shows an example of the traffic violation form designed for the application at hand.

The paper is organized as follow. Section 2 and 3 describes, respectively, system architecture and experimental results. Comments and conclusion are given in section 3.

D. Lopresti, J. Hu, and R. Kashi (Eds.): DAS 2002, LNCS 2423, pp. 66-69, 2002.

(C) Springer-Verlag Berlin Heidelberg 2002 


\section{System Description}

The proposed system is composed of three modules: Preprocessing, extraction of handwritten information, and recognition. Input image is scanned at $300 \mathrm{dpi}$. The output of the scanning process is a black and white image. The preprocessing module uses de-skewing algorithm to correct image inclination and, thereafter, applies thinning process [5] to reduce the image to a one-pixel wide image. Hull [6] described the performance of various image skew detection algorithms. We have evaluated all of the algorithms mentioned in that reference and found the method of Bessho et al [7] is sufficient for our purpose.

As seen in figure 1 the image contains six fields of information, each of which is composed of specific number of squares. The size of all squares are equal (28x28 pixels. For the sake of discussion, we will use the term "infobits" to refer to the information contained in each square. From the point of view of image processing, an infobits is a set of connected black pixels. The marks "- ", localized at specific points, on the right-hand side of the image is included to aid the extraction process. To extract each infobits, we have employed a modified architecture of the Discrete Neural Networks (DNN) proposed by Murshed [8]. The DNN detects one-pixel-wide line segments, and it is composed of slabs whereby each slab is composed of layers of neurons. The size of the slab equals the size of the input image. The modified network consists of one slab only, the size of which equals the size of the square, i.e., $28 \times 28$ pixels. Its purpose is to detect the existence of a square and an infobits. Parallel programming was used to process each neuron simultaneously. A square is detected if $80 \%$ of the boundary pixels are black. This number was determined empirically using database of 4000 images. From the analysis of those images, we have noticed the existence of line discontinuity (number of white pixels in a sequence of adjacent black pixels) due to the scanning and thinning processes. In all cases the magnitude of the discontinuity did not exceed 10 pixels. The extraction network provides two signals, each of which indicates, respectively, the existence of a boundary and an infobits. If the value of each signal is ' 1 ', then the recognition module is triggered and the detected infobits is centralized inside a $28 \times 28$ square, and then passed to the recognition network.

The recognition module is composed of a Probabilistic Fuzzy ART neural network, which is a modified version of the original network introduced by Carpenter and Grossberg [9]. Its internal mechanism is modified such that its output is a probabilistic value that indicates how confidence the network is with respect to the pattern class. The confidence level is set internally. If the network confidence is lower than the preset value, the system requests the user to identify the input pattern. Such user interaction reduces the overall system error to zero. This feature is crucial when dealing with automatic reading of traffic tickets, because a single error means a wrong number plate is ticketed which in turn causes annoyance to its owner.

\section{Experimentation and Results}

The prototype system was evaluated with a database of 8000 images. The database was split into two sets $d_{6000}$ and $d_{2000}$, each of which was used for training/testing and evaluation, respectively. The subscript indicates the magnitude of the data set. The 
magnitude of the training set is $0.8\left|d_{6000}\right|$ and that of the test set is $0.2\left|d_{6000}\right|$. Training and testing were repeated 33 times using the same magnitude of the corresponding data set but different images. It should be noted that the images were selected randomly. The following algorithm describes the experimental setup.

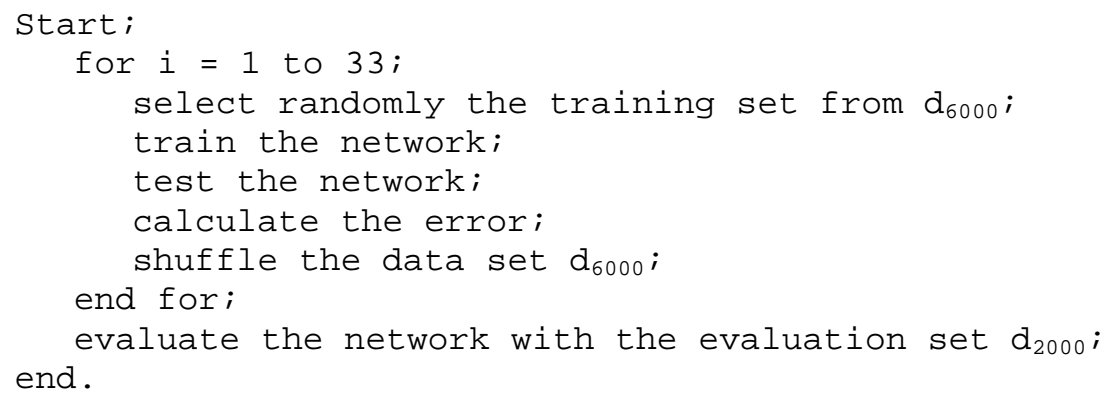

The average correct recognition rate, on the test set, calculated over 33 trials was 93\%. The lowest and highest were, respectively, $87 \%$ and $97 \%$. The recognition rate on the evaluation set was $90 \%$, and the lowest rate was $89 \%$. Note that when applying the confidence criterion and user interaction, the recognition rates increased to $100 \%$.

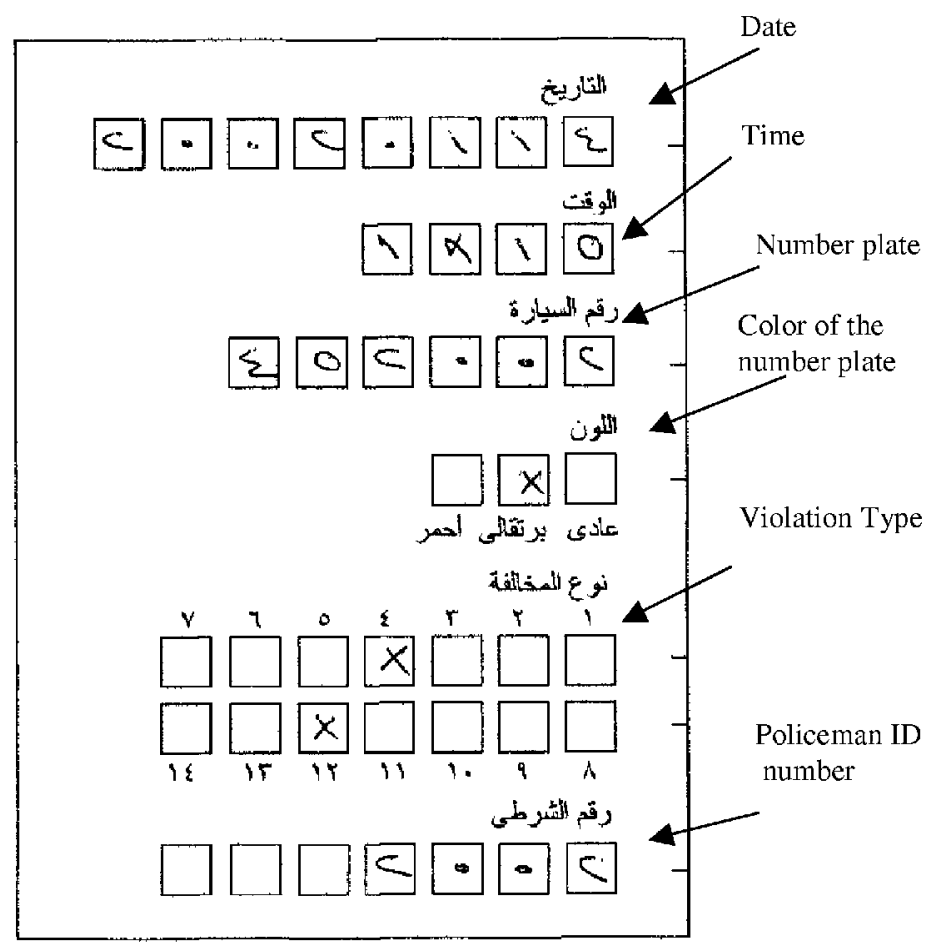

Fig. 1. An image of the traffic ticket form. For legal reasons some information were not shown. Moreover, the number plate and the policeman ID number are fictitious. 


\section{Comments and Conclusion}

In this paper we have presented a prototype system for reading the traffic tickets. The system presented satisfactory results and the application of the confidence criterion helped increasing the recognition rates to $100 \%$. Currently we are working towards improving the recognition rate, which will require fewer interactions from the user, and reducing the processing time, which is currently 10 seconds per form. We will also modify the experimental setup to select the optimum skew detection and thinning algorithms in terms of processing time and recognition rate.

Besides the Fuzzy Art network, we have also tested the Back-propagation NN, Probabilistic NN, and SOM NN. The Fuzzy ART presented better results. The front end of the system was implemented with Visual basic, and all the processing algorithms in Visual $\mathrm{C}++$.

\section{References}

1. Proceedings of the First IAPR Workshop on Document Analysis Systems. Eds. A. Lawrence Spitz and Andreas Dengel. World Scientific Press. 1995.

2. Document Analysis Systems II , Proceedings of the Second IAPR Workshop on Document Analysis Systems. Eds. Jonathan Hull and Suzanne Taylor. World Scientific Press. 1998.

3. Proceedings of the Third IAPR Workshop on Document Analysis Systems. 1998.

4. Proceedings of the Fourth IAPR Workshop on Document Analysis Systems. Eds. Nabeel Murshed and Adnan Amin. 2000.

5. G. Dimauro, S. Impedovo and G. Pirilo, "A new Thinning Algorithm Based on Controlled Detection of Edge Region," Thinning Methodologies for Pattern Recognition, C. Y. Suen and P. S. P. Wang, World Scientific Press, 1994, 969-986.

6. Jonathan Hull, "Document Image Skew Detection: Survey and Annotation Bibliography," Document Analysis Systems II, Second IAPR Workshop on Document Analysis Systems. Eds. Jonathan Hull and Suzanne Taylor. World Scientific Press. 1998, 40-64.

7. G. Bessho, K. Ejiri and J. F. Cullen, "Fast and accurate skew detection algorithm for a text or a document with straight lines," Proceedings of the SPIE - The International Society for Optical Engineering, Conference on Document Recognition 2181 (February 9-10, 1994), 133-140.

8. Nabeel Murshed, “A Discrete Neural Network for Detection of Line Segments," Proceedings of the Fourth IAPR Workshop on Document Analysis Systems. Eds. Nabeel Murshed and Adnan Amin. 2000.

9. Gail Carpenter and Steven Grossberg, "Fuzzy ART: Fast stable learning and categorization of analog patterns by an adaptive resonance system," Neural Networks, 4, 759-771. 